\title{
Electronic Sentinel Surveillance of Influenza-like IIIness
}

\section{Experience from a pilot study in New Zealand}

Mehnaz Adnan'; Donald Peterkin'; Liza Lopez ${ }^{1}$; Graham Mackereth ${ }^{1}$

${ }^{1}$ Institute for Environmental Science and Research Ltd. New Zealand

\section{Keywords}

Influenza-like IIIness, Public Health, Surveillance, HL7

\section{Summary}

Background: Electronic reporting of Influenza-like illness (elLI) from primary care was implemented and evaluated in three general medical practices in New Zealand during May to September 2015.

Objective: To measure the uptake of elLI and to identify the system's strength and limitations. Methods: Analysis of transactional data from the elLI system; comparative study of influenza-like illness cases reported using manual methods and elLl; questionnaire administered to clinical and operational stakeholders.

Results: Over the study period $66 \%$ of total ILI cases were reported using elLI. Reporting timeliness improved significantly compared to manual reporting with an average of 24 minutes from submission by the clinician to processing in the national database. Users found the system to be userfriendly.

Conclusion: elLI assists clinicians to report ILI cases to public health authorities within a stipulated time period and is associated with faster, more reliable and improved information transfer.

\section{Correspondence to:}

Mehnaz Adnan

Institute for Environment Science and Research

Kenepuru Science Centre

34 Kenepuru Drive, Porirua 5022

New Zealand

E-mail: mehnaz.adnan@esr.cri.nz

\section{Appl Clin Inform 2017; 8: 97-107}

https://doi.org/10.4338/ACI-2016-06-RA-0103

received: July 11, 2016

accepted: November 28, 2016

published: February 1, 2017

Citation: Adnan M, Peterkin D, Lopez L, Mackereth G.

Electronic sentinel surveillance of influenza-like illness: Experience from a pilot study in New Zealand. Appl

Clin Inform 2017; 8: 97-107

https://doi.org/10.4338/ACI-2016-06-RA-0103 


\section{Introduction and Background}

The general practitioner (GP)-based sentinel surveillance system for influenza-like illness (ILI) in New Zealand was established in 1989 as part of the World Health Organization's (WHO) Global Influenza Surveillance Network [1]. The Institute for Environmental Science and Research (ESR) undertakes national surveillance from May to September each year in conjunction with local public health services (PHSs) [2]. Surveillance coordinators in each PHS recruit general practices within their region to participate on a voluntary basis in ILI surveillance. This requires GPs to record each week on a standardized form the number of ILI consultations, the age group for each suspected case and the number of specimens taken [3]. Traditionally, GPs or nurses in participating general practices fill out forms manually and fax them to the ILI surveillance coordinators at the PHS. The ILI coordinators manually collate the information on the number of ILI consultations and specimens collected from each general practice and the aggregated information is faxed each week to a national ILI coordinator at ESR. This system has been operating for many years [2]. However, manual data collection is time consuming [2,4], putting additional pressure on the GP practice and PHS staff during winter, and generates only a limited amount of data i.e. ILI counts for each practice by age group.

New Zealand has one of the world's highest rates of the use of Practice Management Systems among primary care physicians [5]. These systems are used primarily to maintain electronic health records, support integrated patient care through electronic referral of patients to secondary care and to obtain electronic laboratory results [6]. The widespread use of Practice Management Systems provides an opportunity to automate the process of ILI reporting from primary care. For a system to be described as 'supporting electronic sentinel surveillance' the reporting process needs to be supported by information technology for at least part of the workflow. Electronic systems for supporting sentinel surveillance are becoming increasingly commonplace. Liljeqvist et al. applied automatic data extraction techniques from GP records in order to collect data for ILI sentinel surveillance in Australia [7]. In Denmark, where the information technology is accessible to essentially all GPs, experience from implementation of an electronic ILI (eILI) during an influenza pandemic in 2009 demonstrates its timeliness in reporting compared with manual data collection [8]. A United States-based comparative study of eILI and manual sentinel surveillance found that the electronic system reduced the burden on clinical staff of disease reporting, and provided consistent and timely reporting [9]. Turbelin et al. [10] implemented a system which allowed GPs to report ILI cases to a central server using a Java based desktop client application. An evaluation of this system showed better integration with the GP's workflow, more timely data and improved data quality.

ESR, aiming to modernize New Zealand's ILI sentinel surveillance system, has thus developed an eILI system. This system allows GPs to complete an electronic form at the time of patient consultation within their Practice Management System and send it to ESR using an HL7 message [11]. HL7 refers to a set of international standards for the transfer of clinical and administrative data between information systems and is used in New Zealand and worldwide [13, 14]. A pilot study of the eILI system was conducted in three General Practices in the Capital and Coast Region from May to September 2015. The objective of this pilot study was to assess the timeliness and user acceptance of the system. Timeliness was assessed by a comparison of ILI case reports received using the eILI system and ILI cases reported by the manual method, within the same region. User acceptance was assessed by quantitative and qualitative analysis of a survey of end users after the pilot. Here we present the findings of the study.

\section{Methods}

\subsection{Settings}

In New Zealand, PHSs focus on environmental health, communicable disease control, tobacco control and health promotion programs. Many of these services include a regulatory component performed by statutory officers appointed under various statutes, though principally under the Health Act 1956. The pilot was conducted by recruiting three general practices in the Capital and Coast re- 
gion in conjunction with the local PHS. These practices are often overbooked making system adaptability and efficiency a critical factor for reporting ILI cases. The practices participated in the pilot study voluntarily. During the pilot study they reported ILI cases using the electronic system only. Two of the practices had been participating in manual ILI sentinel surveillance for nine years and the third practice for one year. The system users were provided with participation information sheets containing the study protocol and instructions for using the system. A training session was conducted in each practice prior to the pilot where the GPs and nurses learnt how to complete the ILI form and discussed the eILI evaluation protocol.

\section{2 elLI System Overview}

The eILI system was designed to assist GPs in reporting ILI cases at the time of patient consultation and to send the information on these cases to ESR in a secure manner. The system consists of an online ILI form, HL7 messaging framework and an ILI database. The ILI form captures information from the Practice Management System and includes pre-populated patient and provider demographic information from the Practice Management System, consistency checks and an intuitive layout. The user completes the ILI form for a patient presenting with ILI symptoms and sends it via a secure pathway using an HL7 message. The completed form is received electronically and the information is stored within the ILI database which is accessed by the national ILI coordinator at ESR for analysis and reporting. The eILI system has been developed using a standards-based solution that is aligned with national strategy advocated by the Health Information Standard Organization, New Zealand [15]. In addition, this system is based on the available and proven electronic form solution, technology and infrastructure; for example electronic referral from community to secondary care implementation [6]. A detailed description of system development is included in [11].

\subsection{Data Sources and Analytical Methods}

Transactional data with records of all ILI cases for July to August 2015 were extracted from the eILI database. The record also included the date and time that the message was sent from the Practice Management System, the date and time the message information was entered into the eILI database at ESR and information about the specimen associated with the record, if one was collected. We also collected ILI data during the study time period for other practices in the same geographic region using the manual method. The data allowed the analysis of eILI uptake i.e. the number of eILI cases per day and the proportion of ILI reports that were electronic versus manual each week. The data also allowed us to measure the timeliness of the eILI system. Timeliness does not have a well-established definition represented by a mathematical equation [16]. However, for evaluating a surveillance system, the timeliness can be defined in terms of the time taken for the information to become available for disease control [17]. We thus measured timeliness in terms of 'report latency time' as the time taken from submission of the ILI form in the Practice Management System until it was sent to the message broker and processed in the ILI database at ESR.

An anonymous questionnaire was designed to collect both qualitative and quantitative data on the acceptability of the technology from the clinical user's perspective. At the completion of the pilot study, users were asked to complete a hard copy of the questionnaire. The first question asked the user to rate the ease of use of the system with the following options: 'very difficult', 'somewhat difficult' or 'not difficult at all'. The other questions evaluated the system using yes/no options for the following key items: time saving, efficiency and cost effectiveness. Responses were treated as unknown if the user did not mark yes or no or if they wrote ' $n / a$ '. Free text fields were provided to allow users to explain the reason for their answer. Users were also asked to provide comments on any challenges, possible system modifications or any other issues or concern they had about the system. The national ILI coordinator provided feedback on whether or not the system was beneficial for surveillance, improved the quality of data, saved time, or reduced operating cost. In addition, the ILI coordinator was asked to provide comments about any further modification needed in the system. The aim was to identify the strengths and limitations of the system.

Data was stored and analyzed using Microsoft Excel version 2013. The time difference between when the ILI report was created in the Practice Management System and the message received and 
processed at ESR was calculated. Cumulative frequency counts were made for cases reported by the eILI process and for the cases reported by the manual process. Forced choice questions were analyzed via descriptive statistical analyses. Open-ended comments for each question were combined to create one note per question and analyzed using data driven thematic content coding [18]. The free text comments and feedback from the national ILI coordinator were segmented into sentences or clauses, with each segment representing one idea which was assigned to a descriptive label. Whenever possible, the label was selected from those already existing; otherwise, a new label was created. Then labels were arranged into a two-level hierarchical scheme with three branches: Application, System and Business. For example 'business' had a sub hierarchy of: extra work, test order, cost saving, beneficial for surveillance, reduced operating cost, and system enhancement. The two-level hierarchy allowed us to interpret data at a deeper level and provide actionable recommendations for future implementation of the system.

\section{Results}

\subsection{Analysis of transactional data Uptake}

- Table 1 shows the total patients enrolled in the practices, the total cases reported and the number of specimens taken for practices that participated in eILI and in manual sentinel surveillance. Three practices participated in each of the eILI and manual systems with a total of 12691 and 13317 patients respectively. The results show that a total of 206 ILI cases were reported, of which $66 \%$ (136 cases) were reported using the eILI system and $34 \%$ (70 cases) by the manual method. More specimens were taken in eILI practices (34 specimens, $62.9 \%$,) than practices using the manual method (20 specimens, $37.0 \%)$

- Figure 1 shows the frequency of eILI messages received per day. The results show that a minimum of 0 to a maximum of 14 messages were received per day. The trend line shows a linear increase in messages over time.

- Figure 2 shows the number each week of ILI reports created using the eILI system and the number reported manually. The results show that a minimum of 1 and a maximum of 14 ILI cases were reported manually each week, compared to a minimum of 6 and a maximum of 26 ILI cases reported electronically each week. The number of ILI cases reported using the electronic method was greater than the number reported manually in eight out of the nine weeks of the study period.

- Figure 3 shows the weekly number of specimens collected for cases reported using the eILI system and cases reported using the manual system. The results show that a minimum of 1 and a maximum of 4 specimens were collected per week in practices using the manual system, while a minimum of 2 and a maximum of 8 specimens were collected per week in practices using the eILI system. The number of specimens collected in practices using the eILI system was greater than or equal to the number collected in practices using the manual system in seven out of the nine weeks.

\section{Report Latency Time}

The report latency time ( $>$ Figure 4 ) shows that $71 \%$ of messages were received within 30 minutes, and $98 \%$ within 60 minutes. Only $2 \%$ of messages took longer than 60 minutes to come through from the Practice Management System to ILI data base. The average time for electronic reports to be received was found to be 24 minutes.

\subsection{Questionnaire Analysis}

\section{Quantitative Analysis}

Eleven GPs used the eILI system to report ILI cases at the time of patient consultation and were invited to complete the questionnaire, and 10 of the 11 filled it in. The responses to the quantitative questions are shown in $>$ Figure 5. All users responded to the question about 'ease of use' with "not difficult at all", so we normalized this response to 'yes' and combined this question with the other questions having yes/no options. Five of the 10 respondents considered that the system saved time; 9 
considered the system to be efficient; 4 considered that the system would reduce the cost; and 5 considered that the system did not impose any challenges to reporting ILI cases. On the other hand, 5 of the 10 respondents found that the system did pose some challenges and did not save time in reporting ILI cases, while 5 were unsure whether the system would reduce the cost. The national ILI surveillance coordinator provided positive feedback for all measures: that the system was beneficial for surveillance, improved the quality of data, and reduced operating cost and time.

\section{Thematic Analysis}

The results of thematic analysis of open ended comments are summarized in $>$ Table 2 and include: positive comments on the ease of use, data quality, beneficial for surveillance and reduction of operating cost and time; mixed views about system response time and improved workflow through electronic reporting; and some complaints about the process of ordering laboratory tests. Users' recommendations included providing a mechanism for linking the report form to the laboratory test ordering system. The national surveillance coordinator recommended implementing automatic summaries and reports.

\section{Discussion}

The widespread use of Practice Management Systems in general practice provides an opportunity to implement an eILI system to improve ILI sentinel surveillance, a process that is currently paperbased, slow and collects limited information. The pilot trial of the eILI system resulted in a significant improvement in timeliness of reporting, from weekly reporting to an average of 24 minutes. The results confirm sustained use of the eILI system, with $66 \%$ of ILI cases reported using it. The system was in fact designed to facilitate data entry by the end user of the system [11] which can potentially reduce the time and effort required to complete the form.

In the questionnaire study, all users found the system easy to use, a majority found it to be efficient, and half of the users found it saved time and did not impose any challenges. The commonest response from users who didn't find the system to be time-saving was that 'a manual tally can be quick as well'. However, the system provides detailed information such as sex, ethnicity, age, geographical location, use of anti-virals and the vaccination status of ILI patients which was not recorded in the manual system. This additional information can be useful for a detailed situational analysis on a daily basis in case of an outbreak. For GPs, the use of online forms for ILI reporting was a mixed experience. Some felt that eILI reporting led to less double handling of data, but others considered it to be an extra piece of work during the limited patient consultation time. In future, a data mining approach which can potentially identify ILI patients from electronic medical records in a Practice Management System could potentially save GP's time [19]. However, the data mining approach would lack the case evaluation by medical staff. The procedure for attaching the laboratory order form was found to be cumbersome, mainly because the specimens for ILI sentinel surveillance were sent to a different laboratory (i.e. ESR laboratories) compared to their usual regional laboratory for other clinical specimens. The national ILI coordinator identified a number of positive aspects including improvements in data collection and data quality, time efficiency, workflow, and operating costs.

This study has number of limitations. First, the HL7 messaging standard used in our eILI system is not simple to implement and maintain. However, it has widely been used for health data exchange and to implement interoperable health solutions in New Zealand, for example, for direct reporting of notifiable diseases from laboratories to the national surveillance system [12], and for electronic referrals of patients from primary to secondary care [6]. Moreover, we are planning to test the implementation of Fast Healthcare Interoperability Resources (FHIR) in the future which will allow the use of data models and XML for information capture and transmission [20]. Second, the evaluation study reported here did not directly measure the completeness and quality of eILI reports, or make a cost-benefit assessment based on staff time savings and operating costs. While the precise interaction of the technology and work practice changes is difficult to separate as an effect of eILI implementation, the reduction in reporting time for ILI cases from weekly reporting to an average of 24 minutes should be seen as a major improvement with the potential to impact public health outcomes 
(e.g. real time reporting in case of an outbreak). Similar improvements have been reported from Danish [8] and French [10] implementations of electronic sentinel ILI surveillance systems. The Danish system was found to be timely and facilitated a detailed situational analysis in a 2009 influenza pandemic [8]. In a French Sentinel Influenza Network experience, GPs reported time savings from using a desktop client for case reporting at the time of consultation [10]. As with the French setting, it is important to note that our findings relate to a situation where general practice is the sentinel ILI information gateway, and where there is essentially universal uptake of Practice Management Systems. In addition, the study was limited to a relatively simple regional setting with just three practices using the electronic system and three the manual system, and with one brand of Practice Management System. Further research would be needed to determine what the effects on uptake, efficiency and content would be if the eILI solution evolved to one with a much larger number of participating practices and implemented within all the brands of proprietary Practice Management Systems being used in the country, and also how the system would perform in the event of an outbreak.

\section{Conclusion}

The eILI solution resulted in much more rapid transfer of data for use at a national level for ILI surveillance compared with manual data collection. Users perceived eILI to provide an easier to use and efficient service. These finding should be confirmed in future implementations and efforts made to link these to broader benefits in public health delivery. The implemented system using a HL7 messaging system integrated with Practice Management Systems, along with the results of the pilot study, provide a platform (both technically and in terms of experience and expectations from the user community) that would be useful in rolling out the system at national level for sentinel ILI surveillance in future. The improvements have the potential to add new capability to the surveillance systems by increasing the amount of data received from practices in real time and making the ILI sentinel surveillance process more useful and efficient in a pandemic setting, hence improving the national surveillance capabilities.

\section{Multiple Choice Questions}

When implementing an Electronic Surveillance System, which of the following mostly allow control and prevention activities to be initiated earlier by public health authorities.

a. Timeliness of reporting

b. Quality of data collected

c. Cost effectiveness

d. Integration with clinical systems

When implementing an Electronic Reporting System for GPs, which of the following must have close attention to avoid severe interruption to clinical work.
a. Intuitive layout
b. Minimum data entry from clinicians
c. Cost effectiveness
d. Healthcare messaging standards

\section{Clinical Relevance Statement}

In an environment with the established use of computing in general practice, uptake of an electronic system for ILI sentinel surveillance can see this system overtake the current manual system. Introduction of eILI surveillance into general practice is associated with faster reporting from primary care to public health authorities. eILI may enhance public health surveillance capabilities through timeliness of reporting, reliability and improved data quality. 


\section{Conflicts of Interest}

The authors performed this work under contract to the New Zealand Ministry of Health. The authors have no commercial interest in any of the software products involved in the solution.

\section{Protection of Human Subjects}

This project was determined to be a national public health surveillance programme by the Health and Disability Ethics Committees (HDEC) and therefore outside their scope for review. The data was collected as a part of public health surveillance and approved by Ministry of Health, New Zealand and executed with the permission of local PHS and participating general practices. 


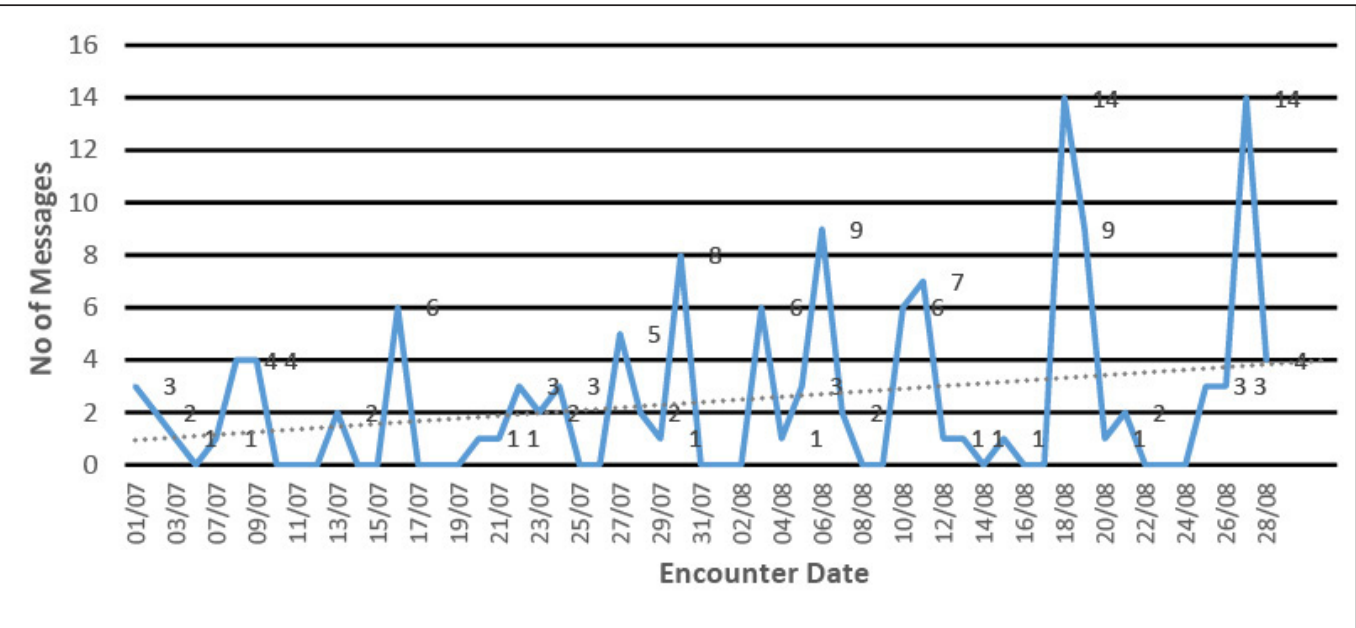

Fig. 1 Frequency of elLI reports received per day

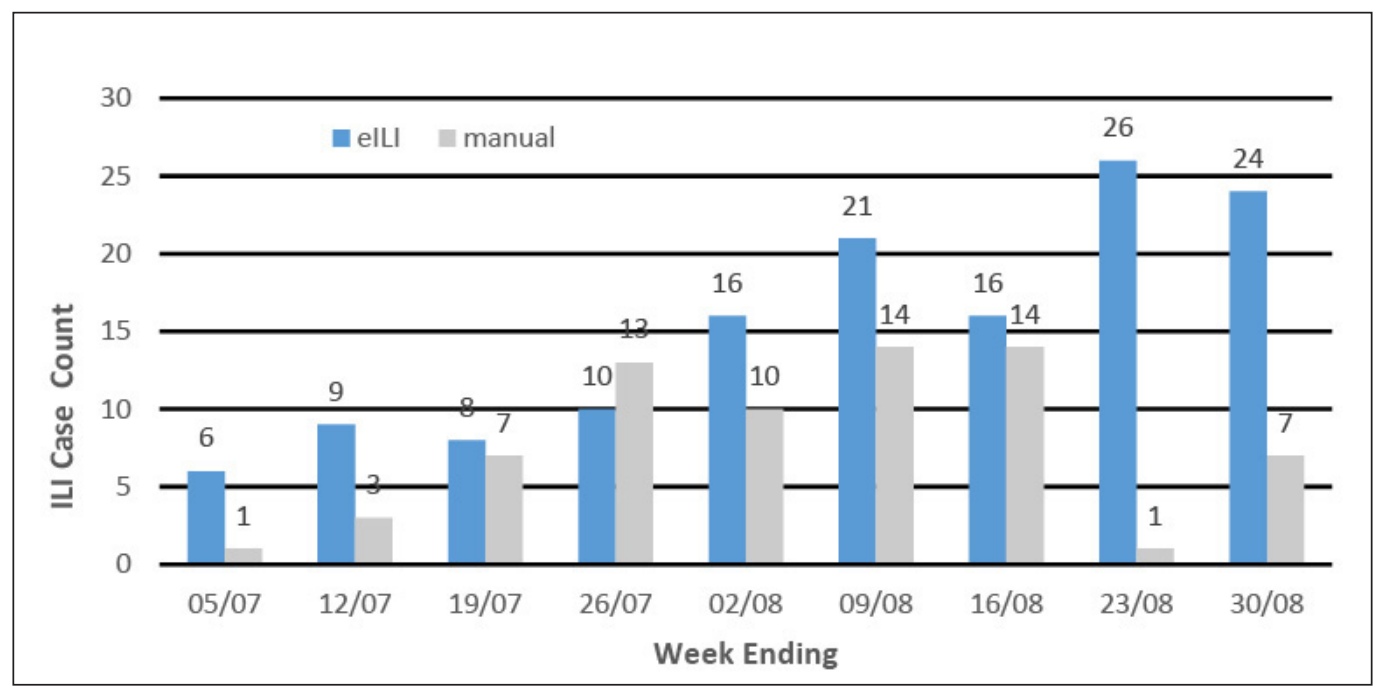

Fig. 2 Frequency of ILI cases notified electronically and manually

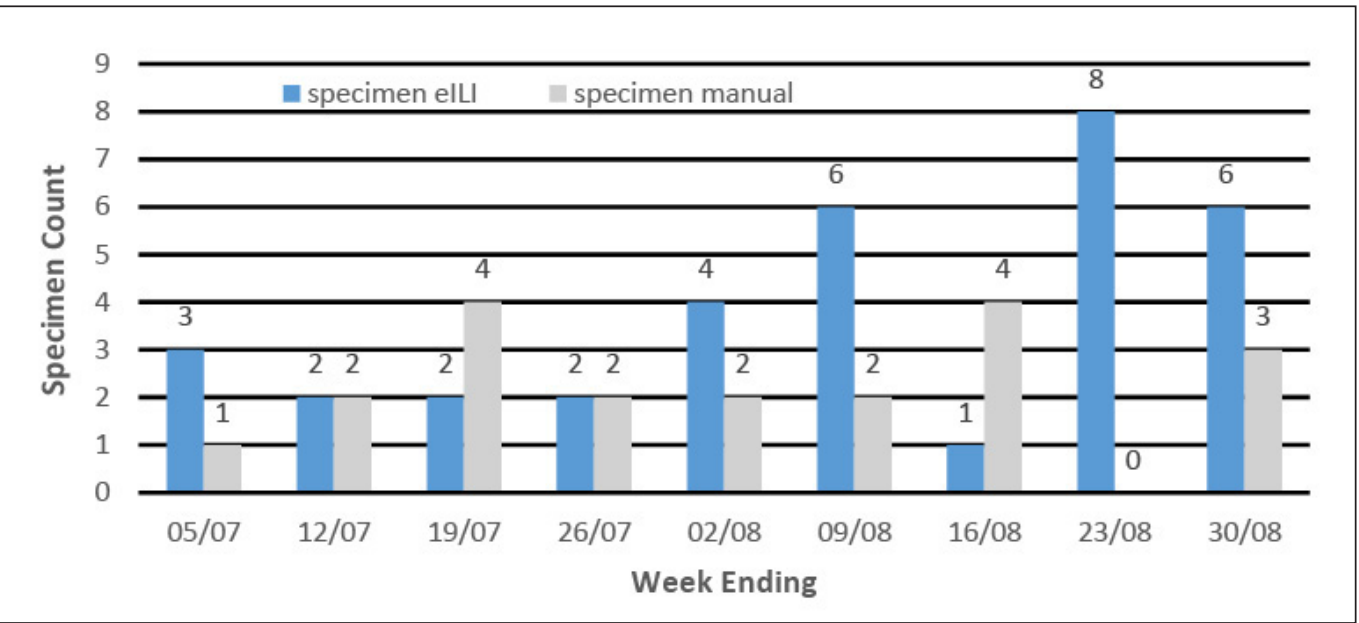

Fig. 3 Frequency of ILI specimen collected in elLI and manual process 


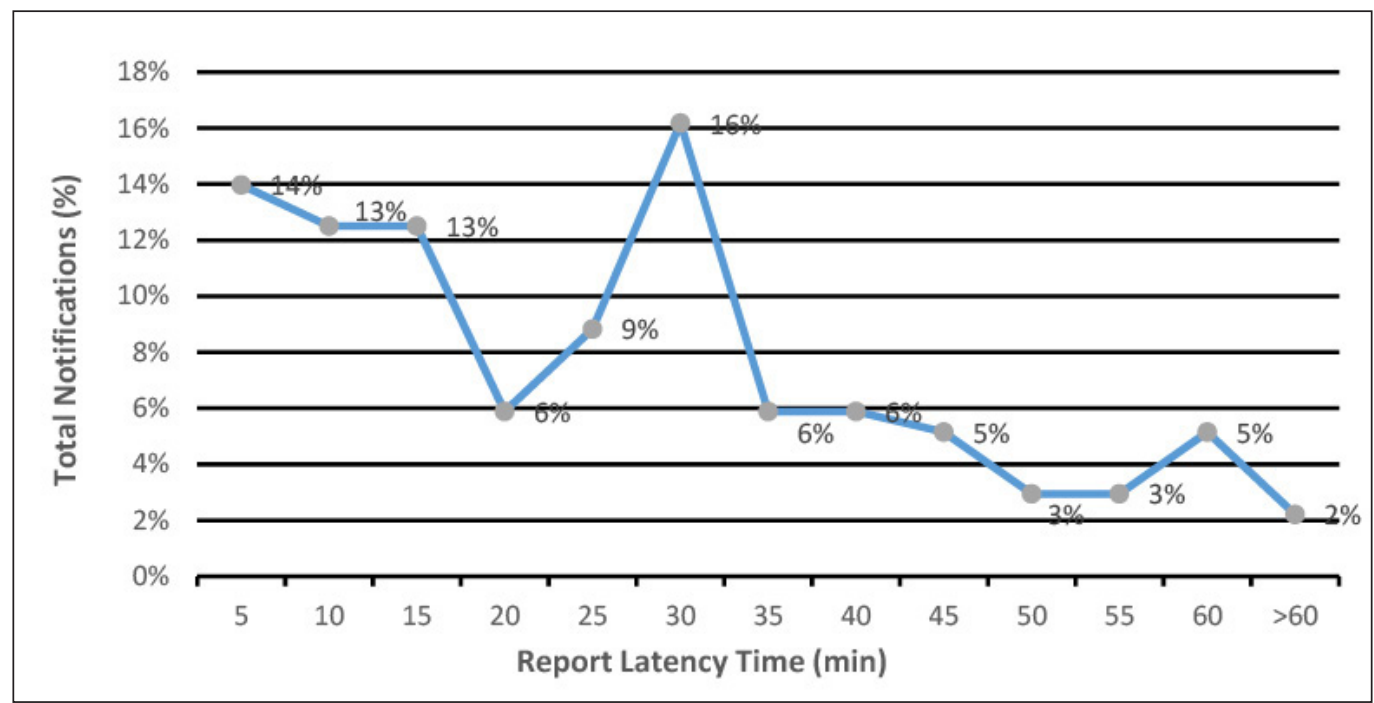

Fig. 4 Time taken by reports to process

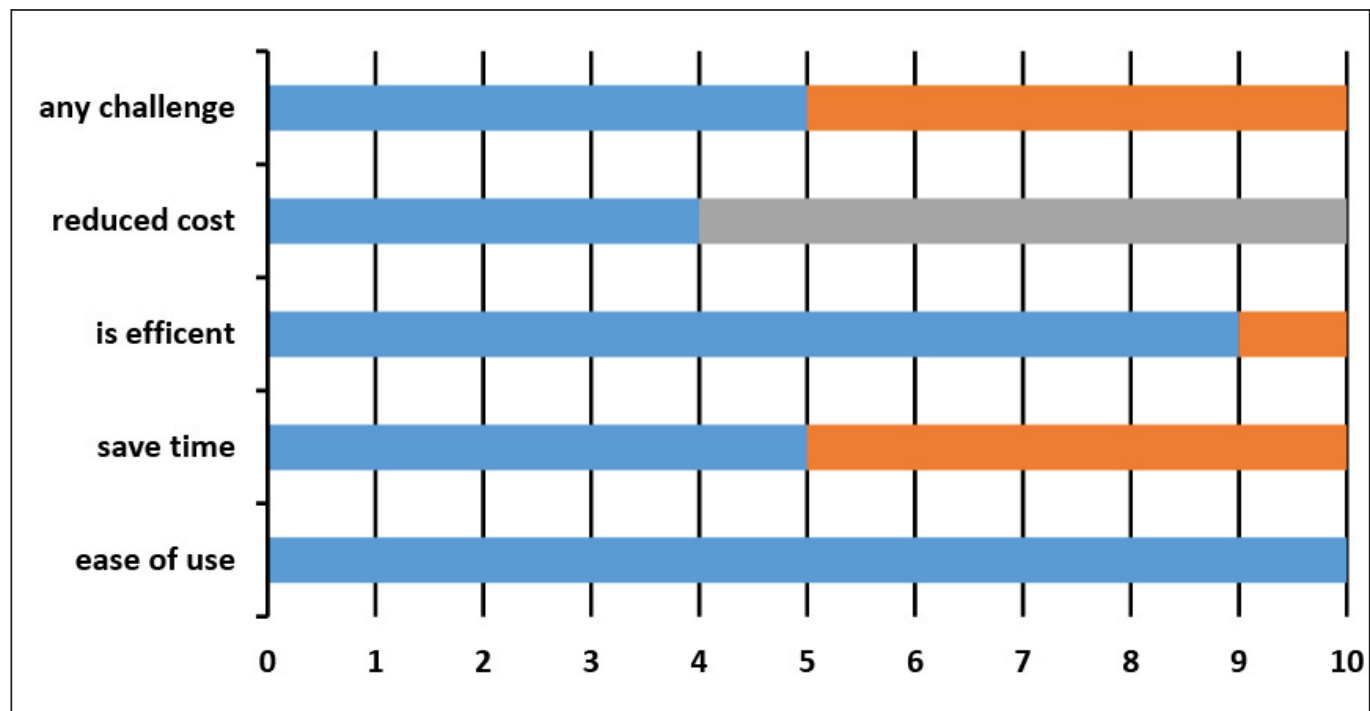

Fig. 5 Responses of forced choice questions

Yes
No
Unknown


Table 1 Statistics of ILI cases reported

\begin{tabular}{|l|l|l|l|l|l|l|l|}
\hline elLI & \multicolumn{9}{|c|}{ Manual } \\
$\begin{array}{l}\text { No of } \\
\text { Practices }\end{array}$ & $\begin{array}{l}\text { Total Patients } \\
\text { Enrolled }\end{array}$ & $\begin{array}{l}\text { Total } \\
\text { Cases }\end{array}$ & $\begin{array}{l}\text { Total } \\
\text { Specimen }\end{array}$ & $\begin{array}{l}\text { No of } \\
\text { Practices }\end{array}$ & $\begin{array}{l}\text { Total Patients } \\
\text { Enrolled }\end{array}$ & $\begin{array}{l}\text { Total } \\
\text { Cases }\end{array}$ & $\begin{array}{l}\text { Total } \\
\text { Specimen }\end{array}$ \\
\hline 3 & 12691 & 136 & 34 & 3 & 13317 & 70 & 20 \\
\hline
\end{tabular}

Table 2 Themes Emerging from Questionnaires/Feedback

\begin{tabular}{|c|c|}
\hline Themes & Key Points \\
\hline Application & $\begin{array}{l}\text { Ease of use: "simple process", straight forward, quick, "inserting duration of illness - should } \\
\text { inform us not to say so many days ( } 8 \text { days) instead to put number only ( } 8 \text { ) } \\
\text { "Data Quality: better than recording manually, improved quality of data: no double handling. } \\
\text { The data as entered by the practice nurse/GPs is sent to ESR. }\end{array}$ \\
\hline System & Response time: faster, PMS slowness \\
\hline Business & $\begin{array}{l}\text { Extra work: "less double handling", "It was an extra piece of admin to do over and above } \\
\text { usual clinical notes" } \\
\text { Test Order: "Printing a copy of the lab form to accompany the specimen was tricky", "Linking } \\
\text { up forms with swabs at ESR end" } \\
\text { Cost Saving: "saves on faxing cost," Unsure as haven't participated in other methods" } \\
\text { Time saving: no manual data entry, no contact the PHU. } \\
\text { Beneficial for surveillance: real time information, no double data entry required, "avoids po- } \\
\text { tential data entry mistakes", scalable, time efficient } \\
\text { Reduced operating cost: "saves data entry time", saves following up time of PHU staff, sur- } \\
\text { veillance coordinator and practices nurses. } \\
\text { System Enhancement: "create reports to summarize the data". }\end{array}$ \\
\hline
\end{tabular}




\section{References}

1. Global Influenza Surveillance and Response System (GISRS). World Health Organisation; Available from: http://www.who.int/influenza/gisrs_laboratory/en/.

2. Influenza Surveillance in New Zealand 2014. Wellington, New Zealand Institute of Environmental Science and Research Ltd (ESR), 201518 June 2015. Available from: https://surv.esr.cri.nz/PDF_surveillance/Virology/FluAnnRpt/Influenzasurveillance2014Final.pdf

3. Huang QS, Bandaranayake D, Lopez LD, Pirie R, Peacey M, Hall R, Bocacao J, Adlam B, Hope V, Croxson M, Basu I. Surveillance for the 2009 pandemic influenza A (H1N1) virus and seasonal influenza virusesNew Zealand, 2009. Morbidity and Mortality Weekly Report 2009; 58(33): 918-921.

4. Dailey L, Watkins RE, Plant AJ. Timeliness of data sources used for influenza surveillance. Journal of the American Medical Informatics Association 2007; 14(5): 626-631.

5. Schoen C, Osborn R, Squires D, Doty M, Rasmussen P, Pierson R, Applebaum S. A survey of primary care doctors in ten countries shows progress in use of health information technology, less in other areas. Health affairs 2012; 31(12): 2805-2816.

6. Warren J, White S, Day KJ, Gu Y, Pollock M. Introduction of electronic referral from community associated with more timely review by secondary services. Applied clinical informatics 2011; 2(4): 546-564.

7. Liljeqvist GT, Staff M, Puech M, Blom H, Torvaldsen S. Automated data extraction from general practice records in an Australian setting: trends in influenza-like illness in sentinel general practices and emergency departments. BMC Public Health 2011; 11(1): 1.

8. Harder KM, Andersen PH, Bæhr I, Nielsen LP, Ethelberg S, Glismann S, Molbak K. Electronic real-time surveillance for influenza-like illness: experience from the 2009 influenza A (H1N1) pandemic in Denmark. Euro Surveill 2011; 16(3): pii-19767.

9. Yih WK, Cocoros NM, Crockett M, Klompas M, Kruskal BA, Kulldorff M, Lazarus R, Madoff LC, Morrison MJ, Smole S, Platt R. Automated Influenza-Like Illness Reporting-An Efficient Adjunct to Traditional Sentinel Surveillance. Public Health Reports 2014; Jan 1: 129(1).

10. Turbelin C, Boëlle PY. Improving general practice based epidemiologic surveillance using desktop clients: the French Sentinel Network experience. Studies in health technology and informatics 2010; 160(Pt 1): 442.

11. Adnan M, Peterkin D, Mackereth G. Development of an Electronic Notification System for Influenza-Like Illness Sentinel Surveillance. In Digital Health Innovation for Consumers, Clinicians, Connectivity and Community: Selected Papers from the 24th Australian National Health Informatics Conference (HIC 2016) 2016; 227: 1.

12. Adnan M, Peterkin D, McLaughlin A, Hill N, editors. HL7 Middleware Framework for Laboratory Notifications for Notifiable Diseases. In Driving Reform: Digital Health is Everyone's Business: Selected Papers from the 23rd Australian National Health Informatics Conference (HIC 2015) 2015; $214: 1$.

13. Tsui FC, Espino JU, Dato VM, Gesteland PH, Hutman J, Wagner MM. Technical description of RODS: a real-time public health surveillance system. Journal of the American Medical Informatics Association 2003; 10(5): 399-408.

14.Edwards JR, Pollock DA, Kupronis BA, Li W, Tolson JS, Peterson KD, Mincey RB, Horan TC. Making use of electronic data: the National Healthcare Safety Network eSurveillance initiative. American journal of infection control 2008; 36(3): S21-S26.

15. New Zealand Ministry of Health. Health Information Standards Organisation. Referrals status and discharges. 2007. Available from: http://healthitboard.health.govt.nz/system/files/documents/ publications/10011-3-rsd-implementation-guide-v2.pdf

16. Stoto MA, Fricker Jr RD, Jain A, Diamond A, Davies-Cole JO, Glymph C, Kidane G, Lum G, Jones L, Dehan K, Yuan C. Evaluating statistical methods for syndromic surveillance. InStatistical Methods in Counterterrorism 2006 (pp. 141-172). Springer New York.

17. Klaucke Klaucke DN, Buehler JW, Thacker SB, Parrish RG, Trowbridge FL, Berkelman RL. Guidelines for evaluating surveillance systems. MMWR Morb Mortal Wkly Rep 1988; 37 (Suppl. 5): 1-8.

18. Joffe H, Yardley L. 4 Content And Thematic Analysis. Research methods for clinical and health psychology. California: Sage 2004; 56-58.

19. MacRae J, Love T, Baker MG, Dowell A, Carnachan M, Stubbe M, McBain L. Identifying influenza-like illness presentation from unstructured general practice clinical narrative using a text classifier rule-based expert system versus a clinical expert. BMC medical informatics and decision making 2015; 15(1): 1 .

20. Bender D, Sartipi K. HL7 FHIR: An Agile and RESTful approach to healthcare information exchange. In Proceedings of the 26th IEEE International Symposium on Computer-Based Medical Systems 2013 Jun 20 (pp. 326-331). IEEE. 